\title{
Quantum Entanglement of a Tunneling Spin with Mechanical Modes of a Torsional Resonator
}

\author{
D. A. Garanin and E. M. Chudnovsky \\ Department of Physics and Astronomy, Lehman College, City University of New York, \\ 250 Bedford Park Boulevard West, Bronx, New York 10468-1589, USA
}

(Received 21 May 2011; published 8 August 2011)

\begin{abstract}
We solve the Schrödinger equation for various quantum regimes describing a tunneling macrospin coupled to a torsional oscillator. The energy spectrum and freezing of spin tunneling are studied. Magnetic susceptibility, noise spectrum, and decoherence due to entanglement of spin and mechanical modes are computed. We show that the presence of a tunneling spin can be detected via splitting of the mechanical mode at the resonance. Our results apply to experiments with magnetic molecules coupled to nanoresonators.
\end{abstract}

DOI: 10.1103/PhysRevX.1.011005

\section{INTRODUCTION}

There has been enormous progress in measurements of individual nanomagnets [1], microcantilevers, and microresonators [2-15]. Experiments have demonstrated that a mechanical torque induced by the rotation of the magnetic moment may be used for developing high-sensitivity magnetic probes and for actuation of microelectromechanical devices. The underlying physics is a direct consequence of the conservation of the total angular momentum: spin plus orbital. While this effect is clear, the mechanism by which the angular momentum of individual spins gets transferred to the rotational motion of a body as a whole is less understood. In a macroscopic body, the mechanism involves complex evolution of interacting spins and phonons toward thermal equilibrium. In contrast, the case of a magnetic nano- or microresonator is simpler due to the great reduction of the number of mechanical degrees of freedom.

Recently, theoretical study of rotating magnetic nanosystems has been conducted within classical [16-19] and semiclassical $[20,21]$ approaches. When spin is treated quantum mechanically, further reduction of the number of degrees of freedom can be achieved when only a few of the low-energy spin states are relevant. In this paper, we consider an effective spin doublet that originates from superposition of a spin-up and a spin-down state. This would be the case, e.g., of a single-molecule magnet. Rigorous quantum-mechanical treatments have been recently suggested for the problem of a tunneling macrospin in a freely rotating body [22] and for the problem of a tunneling macrospin coupled to the rotational modes of a nanoresonator [23]. (See Fig. 1.)

These two problems have one common feature: The spin tunneling becomes suppressed when the body containing the spin is too light. The physics behind this effect is

Published by the American Physical Society under the terms of the Creative Commons Attribution 3.0 License. Further distribution of this work must maintain attribution to the author(s) and the published article's title, journal citation, and DOI.
Subject Areas: Magnetism, Nanophysics, Spintronics

quite clear [24]. Delocalization in the spin space that corresponds to tunneling of spin $\mathbf{S}$ between spin-up and spin-down states reduces the energy by $\Delta / 2$, where $\Delta$ is the splitting of the tunneling doublet. Since spin transitions are accompanied by changes in the orbital (mechanical) angular momentum $L$, they generate rotational motion of the body with energy $\hbar^{2} L^{2} /(2 I)$, where $I$ is the moment of inertia and $L$ is generally of order $S$. At small $I$, such rotations cost so much energy that the tunneling in the ground state becomes frozen. This effect is conceptually similar to the decoherence and freezing of the tunneling of a particle in a double-well potential due to dissipation [25].

In this paper, we provide a fully quantum-mechanical treatment of a macrospin coupled to a mechanical nanoresonator (see Fig. 1) by solving numerically, and where possible analytically, the corresponding Schrödinger equation. By considering various ranges of parameters of the nanoresonator, we reproduce all previously obtained quantum and semiclassical results and establish connection with the problem of a macrospin in a freely rotating body. Qualitatively different behavior observed for different ranges of parameters is described in detail in the following sections. We show that the way to look for these effects is to study the electromagnetic response of the system depicted in Fig. 1. Magnetic susceptibility and the noise spectrum, as well as the time evolution and decoherence

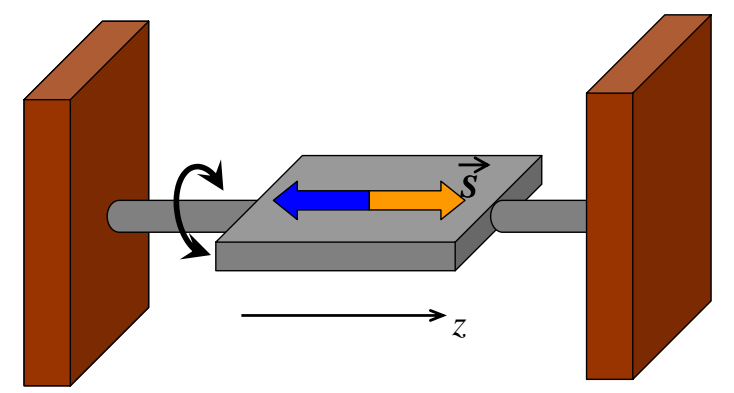

FIG. 1. System studied in the paper. Macrospin (e.g., a magnetic molecule) is attached to a torsional oscillator such that the magnetic anisotropy (quantization) axis is parallel to the axis of mechanical rotations. 
due to entanglement of spin and mechanical modes, are computed and analyzed below. We find that the coupling of a tunneling spin to a mechanical resonator can renormalize tunnel splitting or destroys quantum coherence in molecular-size resonators having very low mechanical frequency. In resonators of greater size, the spin coherence is preserved, and the presence of a tunneling spin can be detected by observing frequency splitting of mechanical oscillations.

\section{THE MODEL}

Consider a model of a tunneling spin $S$, projected onto the lowest tunneling doublet, in a nanoresonator of torsional rigidity $k$ that can rotate around the $z$-axis [21] (see Fig. 1),

$\hat{H}=\frac{\hbar^{2} \hat{L}_{z}^{2}}{2 I_{z}}+\frac{I_{z} \omega_{r}^{2} \hat{\varphi}^{2}}{2}-\frac{W}{2} \sigma_{z}-\frac{\Delta}{2}\left(e^{-2 i S \hat{\varphi}} \sigma_{+}+e^{2 i S \hat{\varphi}} \sigma_{-}\right)$.

Here $\hat{L}_{z}=-i \partial_{\hat{\varphi}}$ is the operator of the mechanical angular momentum, $I_{z}$ is the moment of inertia of the resonator, $\omega_{r}=\sqrt{k / I_{z}}$ is the fundamental frequency of torsional oscillations, $W=2 S g \mu_{B} B_{z}$ is the energy bias due to the longitudinal field $B_{z}, \Delta$ is the tunnel splitting of spin-up and spin-down states due to the crystal field, and $\sigma_{+}$ and $\sigma_{-}$are Pauli matrices. As we shall see below, the behavior of such a system depends on two dimensionless parameters:

$$
\alpha=\frac{2 \hbar^{2} S^{2}}{I_{z} \Delta}, \quad r=\frac{\omega_{r}}{\Delta} .
$$

The limit $\omega_{r}=0$ corresponds to the case where the system consists of a free nanomechanical body (referred to below as a "particle") and a tunneling spin [22]. In this case, the total angular momentum of the system with respect to the $z$-axis is conserved: $J_{z}=S_{z}+L_{z}=$ const. Tunneling of the spin changes $S_{z}$ by $2 S$, and this change is absorbed by the opposite change of $L_{z}$. Thus tunneling occurs between two quantum states having the same total angular momentum eigenvalue $J$. Computation of the eigenstates of the system reduces to the diagonalization of a $2 \times 2$ matrix. The resulting spectrum of the system has the ground state with $J=0$ for [22]

$$
\alpha \leq \alpha_{1}=\left[1-1 /(2 S)^{2}\right]^{-1}
$$

(heavy particle) that corresponds to the spin tunneling between up and down, with the change in the angular momentum absorbed by the rotation of the particle. However, for $\alpha>\alpha_{1}$, the ground state becomes degenerate, and, in the limit $\alpha \gg \alpha_{1}$ (light particle), it approaches $J= \pm S$, which means that the spin cannot tunnel.

In the case where the nanoresonator rotates in an oscillatory fashion under a restoring torque, which is the subject of this work, the total angular momentum of the spin and the mechanical oscillator is no longer conserved. Conservation of the angular momentum occurs in a larger closed system. Still, through the crystal field, tunneling of the spin generates a mechanical torque acting on the torsional oscillator $[21,26]$. This interaction can significantly reduce the spin tunneling for both small and large $r$ when the oscillator is light (large $\alpha$ ). In particular, for small $r$ and large $\alpha$ (see below), the ground state is nondegenerate, but the gap between the ground state and the first excited state becomes so small that the tunneling becomes effectively frozen, similar to the results of Ref. [22].

To solve the quantum-mechanical problem of a spin tunneling in a rotationally oscillating body, it is convenient to use the basis that is a direct product of the two-state "up/down" basis for the spin and the harmonic oscillator basis for the body. Thus we write the system's wave function $|\Psi\rangle$ in the form

$$
|\Psi\rangle=\sum_{m=0}^{\infty} \sum_{\sigma= \pm 1} C_{m \sigma}|m\rangle|\sigma\rangle .
$$

The coefficients $C_{m \sigma}$ satisfy the Schrödinger equation

$$
i \hbar \frac{d C_{m \sigma}}{d t}=\sum_{n=0}^{\infty} \sum_{\sigma^{\prime}= \pm 1} H_{m \sigma, n \sigma^{\prime}} C_{n \sigma^{\prime}}
$$

where

$$
\begin{aligned}
H_{m \sigma, n \sigma^{\prime}}= & E_{m \sigma} \delta_{m n} \delta_{\sigma \sigma^{\prime}} \\
& -\frac{1}{2} \Delta_{\mathrm{eff}}\left(\kappa_{m n} \delta_{\sigma,-1} \delta_{\sigma^{\prime}, 1}+\kappa_{m n}^{*} \delta_{\sigma, 1} \delta_{\sigma^{\prime},-1}\right)
\end{aligned}
$$

are matrix elements of the Hamiltonian, Eq. (1). Here

$$
\Delta_{\text {eff }}=\Delta e^{-\lambda^{2} / 2}
$$

with

$$
\lambda=\sqrt{\frac{2 \hbar S^{2}}{I_{z} \omega_{r}}}=\sqrt{\frac{\alpha}{r}}
$$

whereas

$$
E_{m \sigma}=\hbar \omega_{r}(m+1 / 2)-(1 / 2) W \sigma
$$

are energies in the absence of tunneling and [23]

$$
\kappa_{m n}=(i \lambda)^{m-n} \sqrt{\frac{n !}{m !}} L_{n}^{(m-n)}\left(\lambda^{2}\right)
$$

for $m \geq n$ and a similar expression with $m \leftrightharpoons n$ for $n \geq m$, where $L_{n}^{(m-n)}$ are generalized Laguerre polynomials and $\lambda$ is given by Eq. (8). In particular,

$$
\kappa_{00}=1, \quad \kappa_{10}=\kappa_{01}=i \lambda, \quad \kappa_{11}=1-\lambda^{2} .
$$

For $r \gg 1$, only the ground state of the resonator is relevant in the spin tunneling problem because energies of all other states are too high compared to $\Delta$. In this case, considered in Ref. [23], one returns to a two-state model for the spin with the effective splitting (7). 
For small $r$, the spin couples to many oscillator states, which results in spin decoherence. In this case, one has to diagonalize a large matrix.

\section{ENERGY SPECTRUM AND STATIC SUSCEPTIBILITY}

Setting $C_{m \sigma} \Rightarrow C_{m \sigma} e^{-i(E / \hbar) t}$ in Eq. (5) results in the stationary Schrödinger equation that can be diagonalized numerically to find energy eigenvalues $E_{\mu}$. The results for the distance $\Delta E$ between the ground state and the first excited state as a function of $\alpha$ for different $r$ and $W=0$ are shown in Fig. 2. For $r \ll 1$ and $\alpha>1$, the ground state becomes quasidegenerate with very small, although nonzero, $\Delta E$. This corresponds to the localization of the spin in either spin-up or spin-down state. $\Delta E$ does not exclusively characterize the spin but also contains information about the resonator. Indeed, in the regime where $r<1$ and $\alpha \rightarrow 0$, the spin and the resonator effectively decouple, and $\Delta E \rightarrow \hbar \omega_{r}$, which is the mode of the resonator. In the regime where $r>1$ and $\alpha \rightarrow 0$, one has $\Delta E \rightarrow \Delta$, which corresponds to the spin tunneling mode.

The spin susceptibility is

$$
\chi=\frac{\partial\left\langle\sigma_{z}\right\rangle}{\partial W}
$$

in the limit $W \rightarrow 0$. For a spin in a massive (nonrotating) body, one has

$$
\left\langle\sigma_{z}\right\rangle=\frac{W}{\sqrt{\Delta^{2}+W^{2}}},
$$

and thus the zero-field susceptibility is $\chi_{0}=1 / \Delta$. For a spin in a rotating body, the effective splitting, $\Delta_{\text {eff }}$, can be defined through $\chi=1 / \Delta_{\text {eff }}$, where $\chi=\partial\left\langle\sigma_{z}\right\rangle / \partial W$ follows from the exact numerical diagonalization of the Hamiltonian,

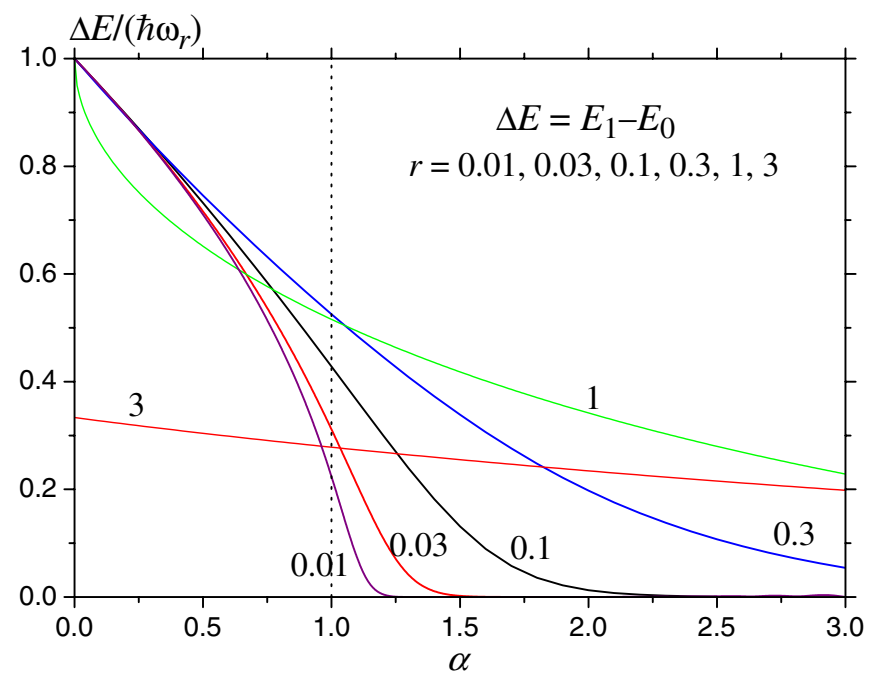

FIG. 2. Distance $\Delta E$ between the ground state and the first excited state as a function of $\alpha$ for different $r$.

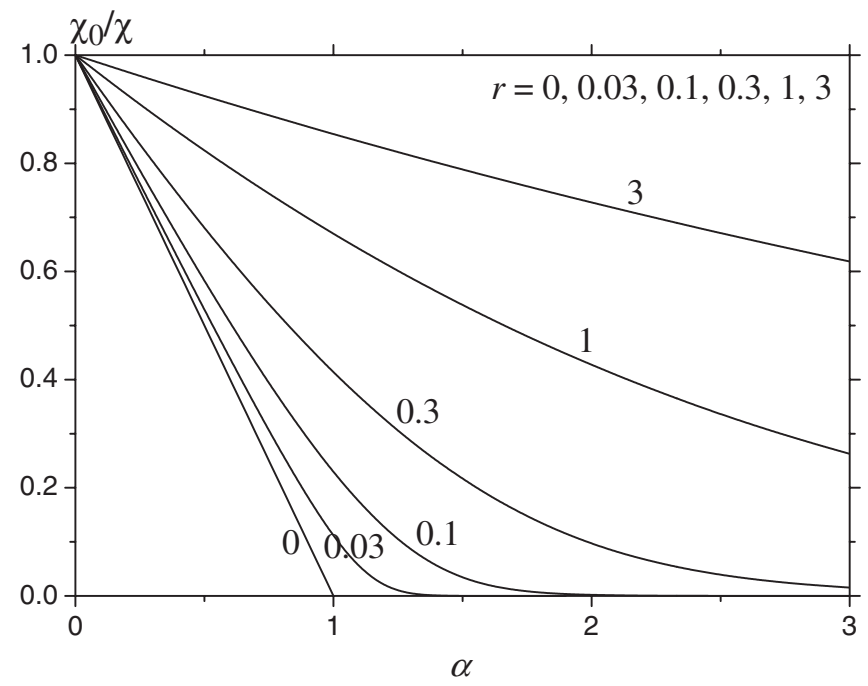

FIG. 3. Reduced inverse susceptibility as a function of $\alpha$ for different $r$.

$$
\left\langle\sigma_{z}\right\rangle=\sum_{m=0}^{\infty} \sum_{\sigma= \pm 1} \sigma\left|C_{0, m \sigma}\right|^{2},
$$

$C_{0, m \sigma}$ being the coefficients of the wave function corresponding to the ground state, $\mu=0$. The dimensionless ratio $\chi_{0} / \chi=\Delta / \Delta_{\text {eff }}$ as a function of $\alpha$ for different $r$ is shown in Fig. 3. For $r \ll 1$ and $\alpha>1$, the zero-field susceptibility becomes very large because of quasidegeneracy of the "up" and "down" spin states. For $r \gg 1$, Eq. (7) is recovered.

\section{SPIN-ROTATION RESONANCE}

The case $\sqrt{\Delta^{2}+W^{2}} \approx \hbar \omega_{r}$ corresponds to the spinrotation resonance that leads to a strong hybridization of spin and rotational states even in the case $\lambda \ll 1$. In the absence of the interaction between the spin and the resonator, $\lambda=0$, the four lowest energy levels are

$$
E=\left\{ \pm \frac{\sqrt{\Delta^{2}+W^{2}}}{2}, \quad \hbar \omega_{r} \pm \frac{\sqrt{\Delta^{2}+W^{2}}}{2}\right\}
$$

where the zero-point energy of the resonator has been dropped. The hybridized levels are $\sqrt{\Delta^{2}+W^{2}} / 2$ and $\hbar \omega_{r}-\sqrt{\Delta^{2}+W^{2}} / 2$. The truncated low-energy Hamiltonian matrix has the form

$$
\mathbb{W}=\left(\begin{array}{cccc}
\frac{W}{2} & 0 & \frac{\Delta}{2} & \frac{\Delta}{2} i \lambda \\
0 & \hbar \omega_{r}+\frac{W}{2} & \frac{\Delta}{2} i \lambda & \frac{\Delta}{2}\left(1-\lambda^{2}\right) \\
\frac{\Delta}{2} & -\frac{\Delta}{2} i \lambda & -\frac{W}{2} & 0 \\
-\frac{\Delta}{2} i \lambda & \frac{\Delta}{2}\left(1-\lambda^{2}\right) & 0 & \hbar \omega_{r}-\frac{W}{2}
\end{array}\right),
$$

where Eq. (11) was used. We look for $E \approx \sqrt{\Delta^{2}+W^{2}} / 2 \approx$ $\hbar \omega_{r} / 2$. Then for $\lambda \ll 1$ the equation $\operatorname{det}(\mathbb{\square}-E \llbracket)=0$ simplifies to 


$$
\left(E-\frac{\sqrt{\Delta^{2}+W^{2}}}{2}\right)\left(E-\hbar \omega_{r}+\frac{\sqrt{\Delta^{2}+W^{2}}}{2}\right)=\frac{\lambda^{2} \Delta^{2}}{4} .
$$

At the resonance, $\hbar \omega_{r}=\sqrt{\Delta^{2}+W^{2}}$, the frequencies of the transition between the ground state $E_{0}=-\sqrt{\Delta^{2}+W^{2}} / 2$ and the closest excited states become

$$
\omega_{ \pm}=\frac{E-E_{0}}{\hbar}=\omega_{r}\left(1 \pm \frac{\lambda}{2} \frac{\Delta}{\sqrt{\Delta^{2}+W^{2}}}\right) .
$$

This formula provides the splitting of the mechanical and spin modes at the resonance. For such a splitting to be observable, the quality factor of the mechanical resonator must exceed $\left(1+W^{2} / \Delta^{2}\right) / \lambda$. Equation (18) can also be obtained within semiclassical approximation [20].

\section{SPIN DYNAMICS}

In the problem of a tunneling spin embedded in a nonrotating crystal, the parameter $\Delta$ has a clear physical meaning as the energy gap between the lowest tunneling doublet. When such a spin is prepared in, e.g., the spinup state at $t=0$, the probability of finding it in the same state at an arbitrary moment of time $t$ oscillates with $t$ according to $\left\langle\sigma_{z}(t) \sigma_{z}(0)\right\rangle=\left\langle\sigma_{z}\right\rangle_{t}=\cos (\Delta t / \hbar)$. When the spin is coupled to a light oscillator and $r \gg 1$, one has only to replace $\Delta$ with $\Delta_{\text {eff }}$. At $r \ll 1$, interaction of the spin with many narrow-spaced modes of the oscillator leads to the decoherence of the spin state.

Spin dynamics is governed by the Schrödinger equation, Eq. (5), and the time dependence of $\left\langle\sigma_{z}\right\rangle$ is given by

$$
\left\langle\sigma_{z}\right\rangle_{t}=\sum_{m=0}^{\infty} \sum_{\sigma= \pm 1} \sigma\left|C_{m \sigma}(t)\right|^{2},
$$

where $C_{m \sigma}(t)$ can be expanded over the eigenstates $C_{\mu ; m \sigma}$ as

$$
C_{m \sigma}(t)=\sum_{\mu} a_{\mu} \exp \left(-\frac{i E_{\mu} t}{\hbar}\right) C_{\mu ; m \sigma},
$$

with the coefficients $a_{\mu}$ being determined by the initial condition. If at $t=0$ the spin was in the "up" state and the particle was in its ground state, one has $a_{\mu}=C_{\mu ; 01}^{*}$. Combining these formulas yields the time dependence

$$
\left\langle\sigma_{z}\right\rangle_{t}=\sum_{\mu \mu^{\prime}} A_{\mu \mu^{\prime}} \exp \left(i \frac{E_{\mu}-E_{\mu^{\prime}}}{\hbar} t\right),
$$

where

$$
A_{\mu \mu^{\prime}}=a_{\mu}^{*} a_{\mu^{\prime}} \sum_{m=0}^{\infty} \sum_{\sigma= \pm 1} C_{\mu ; m \sigma}^{*} \sigma C_{\mu^{\prime} ; m \sigma}
$$

The Fourier spectrum of this time dependence, $2\left|A_{\mu \mu^{\prime}}\right|$, gives the imaginary part of the susceptibility. Plotted as a function of $\hbar \omega_{\mu \mu^{\prime}}=E_{\mu}-E_{\mu^{\prime}}$, it gives an idea of the resonance absorption of the AC field by the spin. For $r \ll 1$ the Fourier spectrum consists, in general, of many lines.

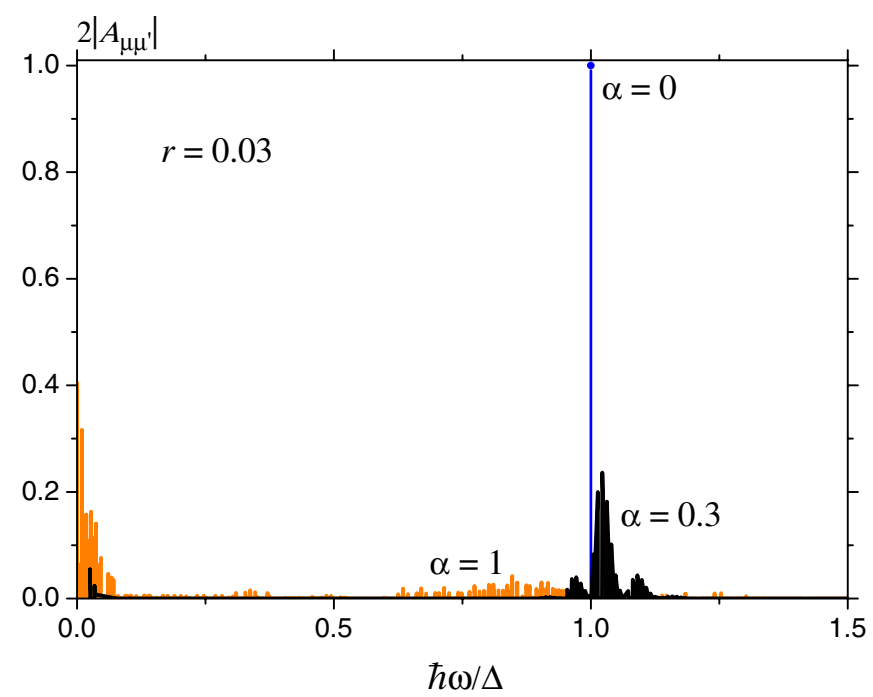

FIG. 4. Fourier spectrum of $\left\langle\sigma_{z}\right\rangle_{t}$ for $r=0.03$ and $\alpha=0,0.3$, and 1 .

In the limit $\alpha \rightarrow 0$, the spin and the torsional oscillator decouple; in this case, only one line of height 1 remains. For $\alpha \ll 1$, there is a narrow group of lines with a spread that gives rise to spin decoherence due to interaction of the spin with the oscillator. At $\alpha>1$, decoherence becomes very strong, and the low-frequency part of the Fourier spectrum corresponding to mechanical oscillations becomes large. These results are shown in Fig. 4 for $r=0.03$.

At $r \gg 1$, there is only one line of height 1 at $\hbar \omega=\Delta_{\text {eff }}$ with $\Delta_{\text {eff }}$ given by Eq. (7). This is natural because in this limit the problem is described by an effective two-state model.

For $r=1$ and small $\alpha$, there is a doublet of lines around $\hbar \omega=\Delta$ because of the resonance interaction between the spin and the resonator; see Eq. (18).

To achieve a better understanding of the spin dynamics, including localization of the spin, one should analyze the time dependence of the spin average shown in Fig. 5. As seen in the figure, for $\alpha=2$, the tunneling is frozen and the spin points in one direction, which is in accordance with Fig. 2. Fast oscillations with a frequency close to $\Delta$ tend to disappear and reappear with time due to the coupling of the spin to a quasicontinuum of the oscillator modes. The recurrence of the oscillations (which is not seen for $r=0.01$ within the plotted time interval) reflects the fact that the oscillator modes are not fully continuous. Fast damped oscillations correspond to the peaks in the Fourier spectrum at $\hbar \omega=\Delta$ in Fig. 4. For $\alpha=0.5$ and 1 in Fig. 5, there are slow oscillations at larger times due to the rotation of the spin together with the nanoresonator. These slow oscillations correspond to the low-frequency peaks in Fig. 4. They occur by virtue of the restoring force that violates conservation of the total angular momentum $J_{z}=L_{z}+S \sigma_{z}$. One can clearly see this in Fig. 6, where fast tunneling oscillations that conserve $J_{z}$ are absent. 

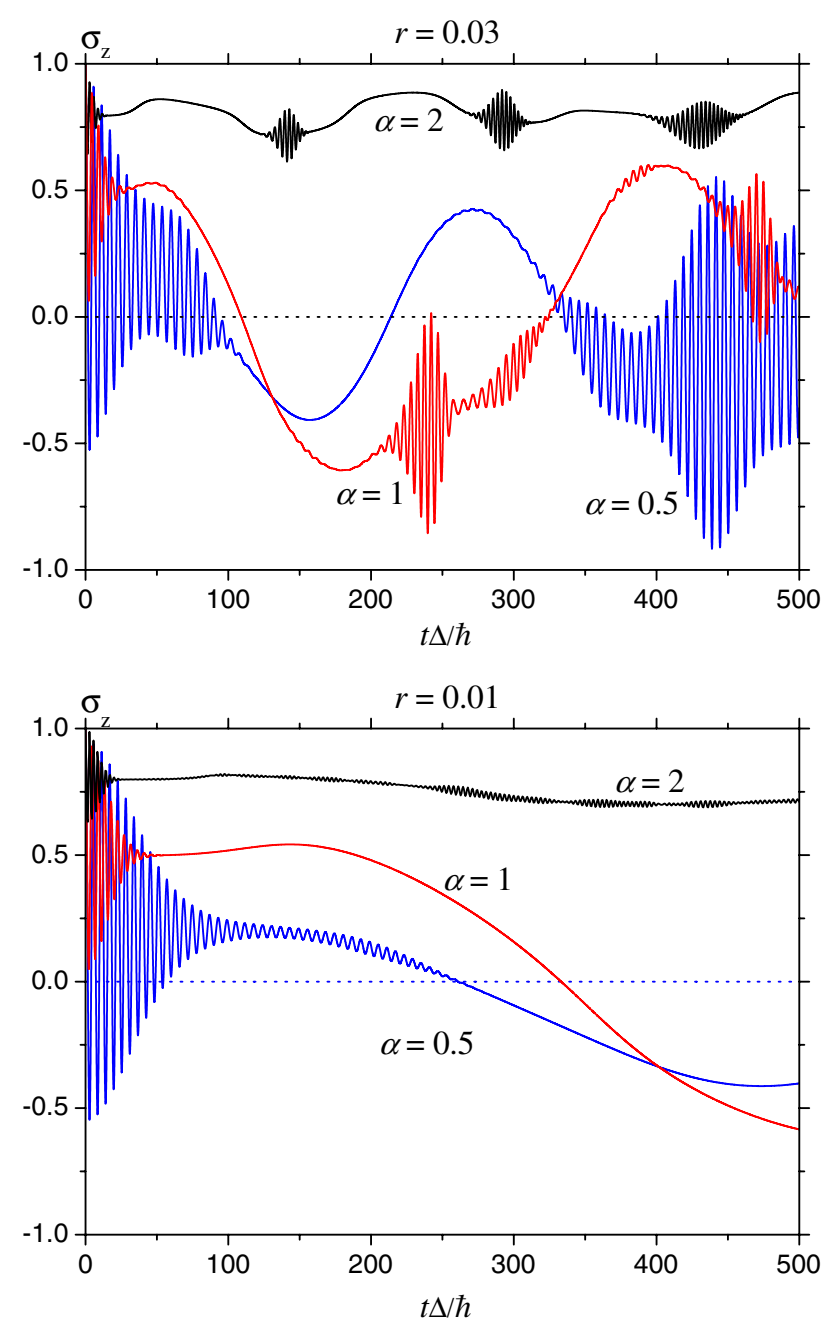

FIG. 5. Time dependences of $\left\langle\sigma_{z}\right\rangle_{t}$ for $r=0.01,0.03$ and $\alpha=$ $0.5,1$, and 2. At $\alpha=2$, the tunneling is frozen and the spin points in one direction.

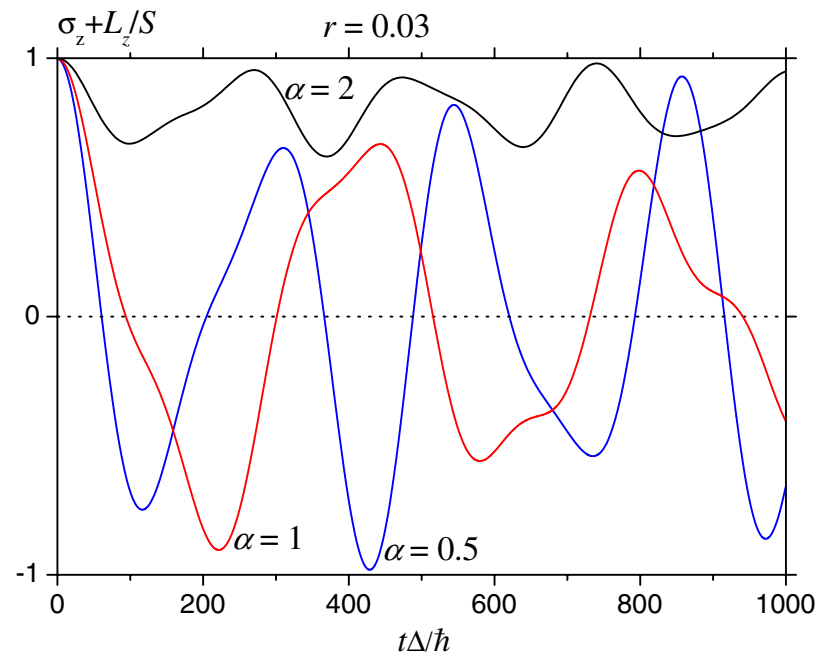

FIG. 6. Time dependence of the total angular momentum. Absence of fast oscillations that conserve $J_{z}$ is apparent.
Finally, it is interesting to compare the dynamics following from the semiclassical method of Refs. [20,21] with the fully quantum theory developed in this paper. The main difference between the two approaches is that the semiclassical method neglects the entanglement while the quantum method takes it into account. The semiclassical approach uses Heisenberg equations of motion for operators $\sigma_{z}$ and $\phi$. In these equations, quantum averages are taken, while quantum correlators between the spin and torsional oscillator are decoupled. The resulting system of equations has the same form as the original Heisenberg equations of motion and looks like classical equations in form. It correctly describes the resonance between the spin and the oscillator, Eq. (18), but it cannot describe the spin decoherence and freezing of tunneling depicted in Fig. 7. The latter effects are, however, generally weak for resonators at and above nanometer size; see below.
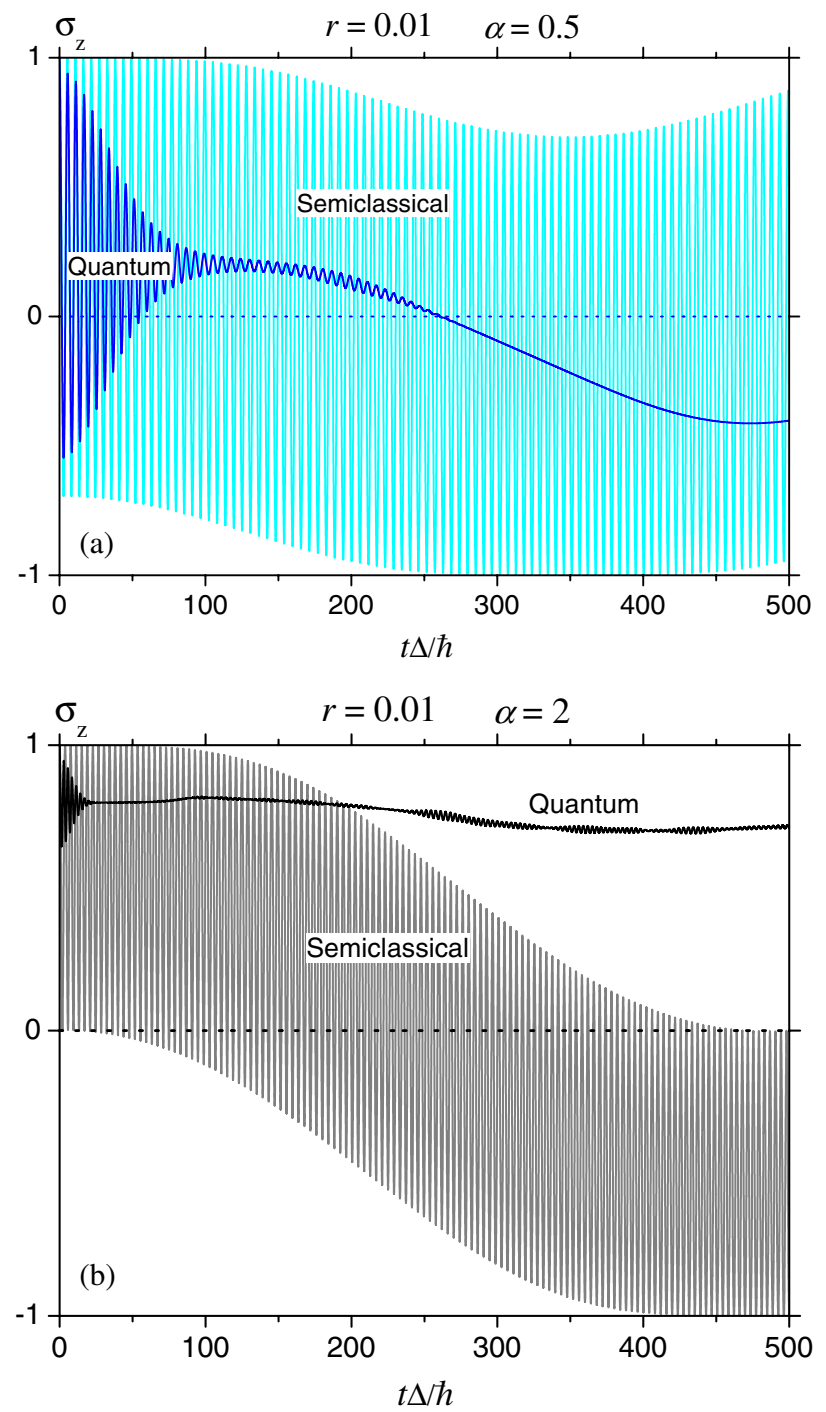

FIG. 7. Time dependences of $\left\langle\sigma_{z}\right\rangle_{t}$ within quantum and semiclassical approaches. In the semiclassical approach, decoherence and freezing of tunneling at $\alpha>1$ [Fig. 7(b)] are absent. 


\section{DISCUSSION}

We have studied energy spectrum, susceptibility, and decoherence in a system consisting of a macrospin rigidly coupled to a torsional mechanical resonator. Our general conclusion is that the coupling does not influence spin tunneling when the resonator is sufficiently large and heavy. However, when one approaches molecular size, the magnetomechanical coupling may lead to strong effects on spin tunneling. Among these effects are freezing of spin tunneling in a free particle or nanoresonator at $\alpha>1$, renormalization of the tunnel splitting for $\lambda>1$ and $r \gg 1$, splitting of the mechanical mode of the resonator under resonance condition $\Delta=\hbar \omega_{r}$, and decoherence of spin oscillations at $r \ll 1$.

To put these statements in perspective, let us consider a magnetic molecule of spin 10 embedded in a torsional resonator in the shape of a paddle of dimensions $20 \times$ $20 \times 10 \mathrm{~nm}^{3}$. As in Ref. [23], we shall assume that the paddle is attached to the walls by two carbon nanotubes of torsional rigidity [27], $k=10^{-18} \mathrm{Nm}$. The moment of inertia of such a system is dominated by the paddle, $I_{z} \sim 10^{-36} \mathrm{~kg} \mathrm{~m}^{2}$, so that $\omega_{r}=\sqrt{k / I_{z}} \sim 10^{9} \mathrm{~s}^{-1}$. The parameter $\lambda$ is then of order $10^{-2}$. For $\Delta / \hbar \ll 10^{9} \mathrm{~s}^{-1}$, coherent spin oscillations at frequency $\Delta / \hbar$ will not be affected by the coupling to the paddle. Decoherence is more likely to be caused by the coupling to nuclear spins or other environmental degrees of freedom in the same manner as for a spin embedded in a macroscopic solid. For $\Delta / \hbar>10^{9} \mathrm{~s}^{-1}$, the parameter $r$ will be small. However, $\alpha$ will be very small compared to 1 , and, thus, in accordance with Fig. 4, no decoherence of spin oscillations due to the coupling with the mechanical oscillations of the paddle will occur in this case, either.

The same will be true even if instead of the paddle one considers, e.g., a $\mathrm{Mn}_{12}$ molecule attached to a carbon nanotube [28]. The relevant moment of inertia is now that of the molecule itself, which for a nanometer size molecule is of order $10^{-42} \mathrm{~kg} \mathrm{~m}^{2}$. The corresponding $\omega_{r}$ is of order $10^{12} \mathrm{~s}^{-1}$ because it scales as $1 / \sqrt{I_{z}}$ (which has not been accounted for in the estimate of Ref. [23]) and $\lambda \sim 0.1$. The two regimes are now $r \gg 1$ for $\Delta / \hbar \ll 10^{12} \mathrm{~s}^{-1}$ and $r \ll 1, \alpha \ll 10^{-2}$ for $\Delta / \hbar \gg 10^{12} \mathrm{~s}^{-1}$. In both limits, the mechanical oscillations should have little effect on coherent spin oscillations with frequency $\Delta / \hbar$. To have a significant effect on the tunnel splitting, one should arrive at $\alpha, \lambda>1$. The condition $\lambda>1$, which leads to a significant renormalization of the tunnel splitting, requires vanishingly small coupling to the walls, $k \ll 10^{-22} \mathrm{Nm}$, as compared to $k=10^{-18} \mathrm{~N} \mathrm{~m}$ for the coupling through a carbon nanotube. For such a choice of $k$, one has $\omega_{r} \ll 10^{10} \mathrm{~s}^{-1}$, and the conditions $\alpha \geq 1$ and $r \ll 1$ needed for significant decoherence are satisfied by $\Delta / \hbar \sim 10^{10} \mathrm{~s}^{-1}$.

The bottom line is that significant effects of spin-rotation coupling on spin tunneling require molecular-size resonators with a vanishing torsional rigidity. Consequently, the freezing of spin tunneling is more difficult to achieve in a mechanical nanoresonator [23] than in a free nanoparticle or free magnetic molecule [22]. The size dependence is easy to understand if one notices that $I_{z} \omega_{r}$ in the expression $\lambda=$ $S \sqrt{2 \hbar / I_{z} \omega_{r}}$ is the measure of the "macroscopicity" of the resonator. Consequently, $I_{z} \omega_{r} \sim \hbar$ needed to achieve large $\lambda$ generally requires a system of the molecular size that is very weakly coupled to the walls. The latter requirement follows from the fact that a very small moment of inertia results in $\omega_{r}=\sqrt{k / I_{z}}$ being large compared to $\Delta / \hbar$ for any reasonable values of $k$ and $\Delta$, thus diminishing the effects of entanglement and decoherence.

For larger resonators, interaction between spin and mechanical degrees of freedom reveals itself only near the resonance. It results in a very interesting quantum phenomenon that can be observed in experiment: splitting of the mechanical mode of the resonator containing a tunneling spin. Remarkably, the splitting obtained within the semiclassical approach $[20,21]$ coincides with the splitting obtained within fully quantum-mechanical treatment. In our example with a paddle having $\lambda \sim 10^{-2}$, the splitting of the mechanical mode at the resonance can be quite significant. For $\Delta<\hbar \omega_{r}$, the resonance will be achieved at $W / \hbar \sim \omega_{r} \sim 10^{9} \mathrm{~s}^{-1}$, which for $S=10$ corresponds to the magnetic field of order $10 \mathrm{G}$. For, e.g., $\Delta / \hbar \sim 10^{8} \mathrm{~s}^{-1}$, according to Eq. (18), this will provide the splitting in the $\mathrm{MHz}$ range that would be possible to observe if the quality factor of the resonator exceeds 1000 . The proposed effect is therefore within experimental reach.

\section{ACKNOWLEDGMENTS}

This work has been supported by the U.S. National Science Foundation through Grant No. DMR-0703639. The authors thank A. A. Kovalev for discussions.

[1] Wolfgang Wernsdorfer, Molecular Magnets: A LongLasting Phase, Nature Mater. 6, 174 (2007).

[2] D. S. Greywall, B. Yurke, P. A. Busch, and C. Arney, LowTemperature Anomalies in the Dissipation of Small Mechanical Resonators, Europhys. Lett. 34, 37 (1996).

[3] E. K. Irish and K. Schwab, Quantum Measurement of a Coupled Nanomechanical Resonator-Cooper-Pair Box System, Phys. Rev. B 68, 155311 (2003).

[4] M. D. LaHaye, O. Buu, B. Camarota, and K. C. Schwab, Approaching the Quantum Limit of a Nanomechanical Resonator, Science 304, 74 (2004).

[5] T. M. Wallis, J. Moreland, and P. Kabos, Generating Single Microwave Photons in a Circuit, Appl. Phys. Lett. 89, 122502 (2006).

[6] A. A. Houck, D. I. Schuster, J. M. Gambetta, J. A. Schreier, B. R. Johnson, J. M. Chow, L. Frunzio, J. Majer, M. H. Devoret, S. M. Girvin, and R. J. Schoelkopf, Generating Single Microwave Photons in a Circuit, Nature (London) 449, 328 (2007). 
[7] M. Poggio, M. P. Jura, C.L. Degen, M. A. Topinka, H. J. Mamin, D. Goldhaber-Gordon, and D. Rugar, An Off-Board Quantum Point Contact as a Sensitive Detector of Cantilever Motion, Nature Phys. 4, 635 (2008).

[8] C. A. Regal, J. D. Teufel, and K. W. Lehnert, Measuring Nanomechanical Motion with a Microwave Cavity Interferometer, Nature Phys. 4, 555 (2008).

[9] A. Jander, J. Moreland, and P. Kabos, Angular Momentum and Energy Transferred Through Ferromagnetic Resonance, Appl. Phys. Lett. 78, 2348 (2001).

[10] J. Moreland, Micromechanical Instruments for Ferromagnetic Measurements, J. Phys. D 36, R39 (2003).

[11] M. Chabot and J. Moreland, Micrometer-Scale Magnetometry of Thin $\mathrm{Ni}_{80} \mathrm{Fe}_{20}$ Films Using Ultrasensitive Microcantilevers, J. Appl. Phys. 93, 7897 (2003).

[12] L. Gao, D. Q. Feng, L. Yuan, T. Yokota, R. Sabirianov, S. H. Liou, M. D. Chabot, D. Porpora, and J. Moreland, A Study of Magnetic Interactions of $\mathrm{Ni}_{80} \mathrm{Fe}_{20}$ Arrays Using Ultrasensitive Microcantilever Torque, J. Appl. Phys. 95, 7010 (2004).

[13] D. Rugar, R. Budakian, H. J. Mamin, and B. W. Chui, Single Spin Detection by Magnetic Resonance Force Microscopy, Nature (London) 430, 329 (2004).

[14] Ying-Ju Wang, Matthew Eardley, Svenja Knappe, John Moreland, Leo Hollberg, and John Kitching, Magnetic Resonance in an Atomic Vapor Excited by a Mechanical Resonator, Phys. Rev. Lett. 97, 227602 (2006).

[15] J.P. Davis, D. Vick, D. C. Fortin, J. A. J. Burgess, W. K. Hiebert, and M. R. Freeman, Nanotorsional Resonator Torque Magnetometry, Appl. Phys. Lett. 96, 072513 (2010).

[16] Alexey A. Kovalev, Gerrit E. W. Bauer, and Arne Brataas, Magnetovibrational Coupling in Small Cantilevers, Appl. Phys. Lett. 83, 1584 (2003).
[17] Alexey A. Kovalev, Gerrit E. W. Bauer, and Arne Brataas, Nanomechanical Magnetization Reversal, Phys. Rev. Lett. 94, 167201 (2005).

[18] Alexey A. Kovalev, Gerrit E. W. Bauer, and Arne Brataas, Current-Driven Ferromagnetic Resonance, Mechanical Torques, and Rotary Motion in Magnetic Nanostructures, Phys. Rev. B 75, 014430 (2007).

[19] Reem Jaafar, E. M. Chudnovsky, and D. A. Garanin, Dynamics of the Einstein-de Haas Effect: Application to a Magnetic Cantilever, Phys. Rev. B 79, 104410 (2009).

[20] Reem Jaafar and E. M. Chudnovsky, Magnetic Molecule on a Microcantilever: Quantum Magnetomechanical Oscillations, Phys. Rev. Lett. 102, 227202 (2009).

[21] Reem Jaafar, E. M. Chudnovsky, and D. A. Garanin, Single Magnetic Molecule Between Conducting Leads: Effect of Mechanical Rotations, Europhys. Lett. 89, 27001 (2010).

[22] E. M. Chudnovsky and D. A. Garanin, Rotational States of a Nanomagnet, Phys. Rev. B 81, 214423 (2010).

[23] Alexey A. Kovalev, Lorien X. Hayden, Gerrit E. W. Bauer, and Yaroslav Tserkovnyak, Macrospin Tunneling and Magnetopolaritons with Nanomechanical Interference, Phys. Rev. Lett. 106, 147203 (2011).

[24] E. M. Chudnovsky, Conservation of Angular Momentum in the Problem of Tunneling of the Magnetic Moment, Phys. Rev. Lett. 72, 3433 (1994).

[25] A. J. Bray and M. A. Moore, Influence of Dissipation on Quantum Coherence, Phys. Rev. Lett. 49, 1545 (1982).

[26] E. M. Chudnovsky, D. A. Garanin, and R. Schilling, Universal Mechanism of Spin Relaxation in Solids, Phys. Rev. B 72, 094426 (2005).

[27] J. C. Meyer, M. Paillet, and S. Roth, Single-Molecule Torsional Pendulum, Science 309, 1539 (2005).

[28] L. Bogani and W. Wernsdorfer, Molecular Spintronics Using Single-Molecule Magnets, Nature Mater. 7, 179 (2008). 